\title{
THE NOETHER-LEFSCHETZ LOCUS OF SURFACES IN TORIC THREEFOLDS
}

\author{
UGO BRUZZO ${ }^{\S \ddagger}$ AND ANTONELLA GRASSI \\ $\S$ Area di Matematica, Scuola Internazionale Superiore di Studi \\ Avanzati (SISSA), Via Bonomea 265, 34136 Trieste, Italia \\ $\ddagger$ Istituto Nazionale di Fisica Nucleare, Sezione di Trieste \\ ฯ Department of Mathematics, University of Pennsylvania, \\ David Rittenhouse Laboratory, 209 S 33rd Street, \\ Philadelphia, PA 19104, USA
}

\begin{abstract}
The Noether-Lefschetz theorem asserts that any curve in a very general surface $X$ in $\mathbb{P}^{3}$ of degree $d \geq 4$ is a restriction of a surface in the ambient space, that is, the Picard number of $X$ is 1 . We proved previously that under some conditions, which replace the condition $d \geq 4$, a very general surface in a simplicial toric threefold $\mathbb{P}_{\Sigma}$ (with orbifold singularities) has the same Picard number as $\mathbb{P}_{\Sigma}$. Here we define the Noether-Lefschetz loci of quasi-smooth surfaces in $\mathbb{P}_{\Sigma}$ in a linear system of a Cartier ample divisor with respect to a (-1)-regular, respectively 0-regular, ample Cartier divisor, and give bounds on their codimensions. We also study the components of the Noether-Lefschetz loci which contain a line, defined as a rational curve which is minimal in a suitable sense.
\end{abstract}

\section{IntRoduction}

The Noether-Lefschetz theorem states that any curve in a very general surface $X$ in $\mathbb{P}^{3}$ of degree $d \geq 4$ is a restriction of a surface in the ambient space, namely the Picard number of $X$ is 1 (a point is very general if it lies outside a countable union of closed subschemes of positive codimension). The Noether-Lefschetz locus is the locus where the Picard number

E-mail address: bruzzo@sissa.it, grassi@sas.upenn.edu.

Date: July 25, 2017.

1991 Mathematics Subject Classification. 14C22, 14J70, 14M25.

Support for this work was provided by the NSF Research Training Group Grant DMS-0636606, the Brazilian CNPq grant 310002/2015-0, PRIN "Geometria delle varietà algebriche," and GNSAGA-INdAM. U.B. is a member of the vBAC group. 
is greater than 1. Green [10, 11] and Voisin [29] proved that if $\mathcal{S}_{d}$ is the locus of the degree $d$ surfaces in $\mathbb{P}^{3}$ whose Picard number is not 1 , every component of $\mathcal{S}_{d}$ has codimension $\geq d-3$, with equality for the components of surfaces containing a line. This result has been generalized by Otwinowska [24] to hypersurfaces in $\mathbb{P}^{n}$; also Lopez-Maclean obtained a bound on the codimension of the Noether-Lefschetz locus of surfaces associated with a very ample line bundle in smooth threefolds, under some conditions [17].

We continue the analysis of [4], and consider a projective toric threefold $\mathbb{P}_{\Sigma}$ with orbifold singularities; $\mathbb{P}_{\Sigma}$ is associated with a 3 -dimensional complete simplicial fan $\Sigma$ and is $\mathbb{Q}$ factorial (see Section 2.1). Let $\beta$ be a nef (numerically effective) class in the class group $C l\left(\mathbb{P}_{\Sigma}\right)$ of Weil divisors modulo rational equivalence and consider a surface $X$ in $\mathbb{P}_{\Sigma}$ whose class (degree) in $C l\left(\mathbb{P}_{\Sigma}\right)$ is $\beta$. If $X$ is general, it is quasi-smooth, that is, its only singularities are those inherited from $\mathbb{P}_{\Sigma}$ (Section 2.2).

Let $\mathcal{M}_{\beta}$ be the moduli space of surfaces in $\mathbb{P}_{\Sigma}$ of degree $\beta$ modulo automorphisms of $\mathbb{P}_{\Sigma}$. In [4], see Theorem 4.2 in Section 4, we proved that for $\beta$ ample and $-\beta_{0}$ the canonical class of $\mathbb{P}_{\Sigma}$, if the multiplication morphism

$$
R(f)_{\beta} \otimes R(f)_{\beta-\beta_{0}} \rightarrow R(f)_{2 \beta-\beta_{0}}
$$

is surjective, very general points of $\mathcal{M}_{\beta}$ correspond to surfaces whose Picard number equals the Picard number of $\mathbb{P}_{\Sigma}$; here $R(f)$ is the Jacobian ring, see Section 2.2. If $\mathbb{P}_{\Sigma}=\mathbb{P}^{3}$ and $\beta=d \geq 4$, or equivalently $\beta-\beta_{0}$ is nef, the morphism in (11) is always surjective. Also, the morphism is surjective whenever $\beta-\beta_{0}$ is trivial, that is, $X$ is a K3 surface in a Fano threefold $\mathbb{P}_{\Sigma}$. If $\mathbb{P}_{\Sigma}$ is an Oda variety, that is, the sum of two polytopes associated with a nef and an ample divisor is equal to their Minkowski sum, the multiplication map in (1) is always surjective, see Section 3.2 .

If we write $\beta-\beta_{0}=n \eta$ for an ample Cartier primitive class $\eta$, the condition $n \geq 0$ generalizes the classical condition $d \geq 4$. We define the Noether-Lefschetz locus with respect to $\beta$ to be the closed subscheme $U_{\eta}(n)$ of $\mathcal{M}_{\beta}$ corresponding to quasi smooth surfaces whose Picard number is strictly larger than that of $\mathbb{P}_{\Sigma}$ (Definition 4.5). In particular we have an upper bound on the codimension of any irreducible component on the NoetherLefschetz locus:

\section{Proposition 4.6.}

$$
\operatorname{codim} U_{\eta}(n) \leq h^{2,0}(S)=h^{0}\left(\mathbb{P}_{\Sigma}, \mathcal{O}_{\mathbb{P}_{\Sigma}}(n \eta)\right),
$$

where $S$ is a quasi-smooth surface in the linear system $\beta$. 
The classical proof of the codimension of the Noether-Lefschetz locus for $\mathbb{P}_{\Sigma}=\mathbb{P}^{3}$ relies implicitly on the fact that $\eta=\mathcal{O}_{\mathbb{P}^{3}}(1)$ is $(-1)$-regular and any line bundle of degree $d \geq 4$ is 0 -regular. However, in Theorem 3.8 we show that $\mathbb{P}^{n}$ is the only toric $n$-fold with an ample $(-1)$ regular line bundle. So we consider toric varieties with a 0-regular ample line bundle. Our proof generalizes the arguments of Green in [10, 11] and relies on vanishing theorems and dualities for toric varieties, as well as the Castelnuovo-Mumford regularity of certain bundles.

In Section 4 we bound the codimension of the Noether-Leschetz locus. In Theorem 4.12 we prove

Theorem 4.12, Let $\mathbb{P}_{\Sigma}$ be a simplicial toric threefold, $\eta$ an ample primitive Cartier class, $\beta_{0}=-K_{\mathbb{P}_{\Sigma}}, \beta \in \operatorname{Pic}\left(\mathbb{P}_{\Sigma}\right)$ an ample Cartier class that satisfies $\beta-\beta_{0}=n \eta$ for some $n \geq 0$. Assume that $\beta$ is 0-regular with respect to $\eta$. If $\eta$ is (-1)-regular, then

$$
\operatorname{codim} U_{\eta}(n) \geq n+1 \text {. }
$$

If $\eta$ is 0-regular, then

$$
\operatorname{codim} U_{\eta}(n) \geq n
$$

Corollary 4.13. Let $\mathbb{P}_{\Sigma}$ be a simplicial Fano toric threefold, $\eta$ a primitive nef divisor, $\beta_{0}=-K_{\mathbb{P}_{\Sigma}}, \beta \in \operatorname{Pic}\left(\mathbb{P}_{\Sigma}\right)$ an ample Cartier class that satisfies $\beta-\beta_{0}=n \eta$ for some $n \geq 3$. If $\eta$ is (-1)-regular then

$$
\operatorname{codim} U_{\eta}(n) \geq n+1
$$

If $\eta$ is 0-regular, then

$$
\operatorname{codim} U_{\eta}(n) \geq n
$$

We prove a similar result for Oda varieties in Theorem 4.15,

We consider various examples in Section 4.3, in Section 5 we consider the components of the loci $U_{\eta}(n)$ which contain a line, defined as a rational curve that is "minimal" in a suitable sense (i.e., its intersection with the ample class $\eta$ is 1 ). We show that the codimension of these components is $n+1$, as in the classical case. To do that, we prove a Severi-type vanishing theorem, and, in a singular case, we study the Hilbert schemes of lines. 
Acknowledgements. We thank R. Lazarsfeld, F. Perroni and C. Voisin for useful discussions, and the referee for many helpful suggestions. This paper was partly written while the first author was visiting UFSC in Florianópolis, Brazil, supported by the CNPq grant 310002/2015-0 and the University of Pennsylvania. He gratefully acknowledges CNPq and the Department of Mathematics at UFSC, especially its Geometry group, for the hospitality and the Department of Mathematics at the University of Pennsylvania.

\section{Preliminaries}

2.1. Divisors in toric varieties and the Picard number. Let $M$ be a free abelian group of rank $r$, let $N=\operatorname{Hom}(M, \mathbb{Z})$, and $N_{\mathbb{R}}=N \otimes_{\mathbb{Z}} \mathbb{R}$. Let $\Sigma$ be a rational simplicial complete $r$-dimensional fan in $N_{\mathbb{R}}$. It defines a complete toric variety $\mathbb{P}_{\Sigma}$ of dimension $r$ having only Abelian quotient singularities. $\mathbb{P}_{\Sigma}$ is said to be simplicial, and is an orbifold.

Let $C l\left(\mathbb{P}_{\Sigma}\right)$ be the group of Weil divisors in $\mathbb{P}_{\Sigma}$ modulo rational equivalence, and $\operatorname{Pic}\left(\mathbb{P}_{\Sigma}\right)$ the group of line bundles on $\mathbb{P}_{\Sigma}$ modulo isomorphism; both are finitely generated Abelian groups, and $\operatorname{Pic}\left(\mathbb{P}_{\Sigma}\right)$ is free. Since $\Sigma$ is simplicial, $\mathbb{P}_{\Sigma}$ is $\mathbb{Q}$-factorial, i.e., the natural inclusion $\operatorname{Pic}\left(\mathbb{P}_{\Sigma}\right) \hookrightarrow C l\left(\mathbb{P}_{\Sigma}\right)$ becomes an isomorphism after tensoring by $\mathbb{Q}$. The rank of the two groups is also the Picard number, the rank of the Néron-Severi group of $\mathbb{P}_{\Sigma}$. Recall that the Néron-Severi group of a variety $Y$ is the image of the Picard group in the second cohomology group with integer coefficients. One can define its rank as $\operatorname{dim}_{\mathbb{Q}} N S(Y) \otimes_{\mathbb{Z}} \mathbb{Q}=$ $\operatorname{dim}_{\mathbb{Q}}\left(H^{2}(Y, \mathbb{Q}) \cap H^{1,1}(Y, \mathbb{C})\right)$.

Let $S$ be the Cox ring, that is, the algebra over $\mathbb{C}$ generated by the homogeneous coordinates associated with the rays of $\Sigma$; so $S=\mathbb{C}\left[x_{\rho}\right]$, where $\rho$ runs over the rays of the fan [6]. This is a generalization of the coordinate ring of $\mathbb{P}_{\Sigma}=\mathbb{P}^{n}$, in which case the Cox ring is $\mathbb{C}\left[x_{0}, \cdots, x_{n}\right]$. Recall that each ray $\rho$ determines a Weil divisor $D_{\rho}$; a monomial $\prod x_{\rho}^{a_{\rho}}$ determines a divisor $D=\sum_{\rho} a_{\rho} D_{\rho}$ and $\operatorname{deg}(D)=\operatorname{deg}\left(\prod x_{\rho}^{a_{\rho}}\right) \in C l\left(\mathbb{P}_{\Sigma}\right)$ induces a grading of $S=\oplus_{\gamma \in C l\left(\mathbb{P}_{\Sigma}\right)} S_{\gamma}$.

2.2. Surfaces in $\mathbb{P}_{\Sigma}$. Let $D$ be a nef divisor of class $\beta$ in $C l\left(\mathbb{P}_{\Sigma}\right)$; then $\mathcal{O}_{\mathbb{P}_{\Sigma}}(D)$ is generated by its global sections [18, Th. 1.6]. Let $X$ be a surface in $\mathbb{P}_{\Sigma}$ whose class in $C l\left(\mathbb{P}_{\Sigma}\right)$ is $\beta$; if $X$ is general, it is quasi-smooth, that is, its only singularities are those inherited from $\mathbb{P}_{\Sigma}$ [20, Lemma 6.6, 6.7]. In particular, $X$ is an orbifold if $\mathbb{P}_{\Sigma}$ is simplicial.

Let $f$ be a section of the line bundle $\mathcal{O}_{\mathbb{P}_{\Sigma}}(D)$ such that $X$ is the locus $f=0$. The ideal $J(f)$ is the ideal in $S$ generated by the derivatives of $f$, and the Jacobian ring $R(f)$ is defined as $R(f)=S / J(f)$. It is naturally graded by the class group $C l\left(\mathbb{P}_{\Sigma}\right)$. 
2.3. The dualizing sheaf and vanishing theorems. Let $\mathbb{P}_{\Sigma, 0}$ be the smooth locus of $\mathbb{P}_{\Sigma}$, and let $j: \mathbb{P}_{\Sigma, 0} \rightarrow \mathbb{P}_{\Sigma}$ be the corresponding open immersion. The sheaf $\widehat{\Omega}_{\mathbb{P}_{\Sigma}}^{p}$ of Zariski $p$-forms on $\mathbb{P}_{\Sigma}$ is the sheaf $j_{*} \Omega_{\mathbb{P}_{\Sigma}, 0}^{p}$, where $\Omega_{\mathbb{P}_{\Sigma}, 0}^{p}$ is the sheaf of $p$-differentials on $\mathbb{P}_{\Sigma, 0}$. The sheaf $\omega_{\mathbb{P}_{\Sigma}}=\widehat{\Omega}_{\mathbb{P}_{\Sigma}}^{r}$ is the dualizing sheaf of $\mathbb{P}_{\Sigma}$. It is a reflexive coherent sheaf of rank 1 , and determines a class in $C l\left(\mathbb{P}_{\Sigma}\right)$ that we shall denote $-\beta_{0}$. If $\beta$ is the class of a Weil divisor, we shall denote by $\mathcal{O}_{\mathbb{P}_{\Sigma}}\left(\beta-\beta_{0}\right)$ the sheaf $\mathcal{O}_{\mathbb{P}_{\Sigma}}(\beta) \otimes \omega_{\mathbb{P}_{\Sigma}}$.

If $\beta$ is an ample class in $\operatorname{Pic}\left(\mathbb{P}_{\Sigma}\right)$, the Bott-Danilov-Steenbrink vanishing theorem [22, Ch. 3] says:

$$
H^{q}\left(\mathbb{P}_{\Sigma}, \mathcal{O}_{\mathbb{P}_{\Sigma}}(\beta) \otimes \widehat{\Omega}_{\mathbb{P}_{\Sigma}}^{p}\right)=0
$$

for $1 \leq q \leq r$ and $0 \leq p \leq r$.

Toric Serre duality ([7], Theorem 9.2.10) then implies

$$
H^{q}\left(\mathbb{P}_{\Sigma}, \mathcal{O}_{\mathbb{P}_{\Sigma}}(-\beta) \otimes \widehat{\Omega}_{\mathbb{P}_{\Sigma}}^{p}\right)=0
$$

for $0 \leq q \leq r-1$ and $0 \leq p \leq r$.

If $\beta$ is Cartier and nef, Mavlyutov's vanishing theorem [19] holds since $\mathbb{P}_{\Sigma}$ is complete and simplicial, and one has

$$
H^{q}\left(\mathbb{P}_{\Sigma}, \mathcal{O}_{\mathbb{P}_{\Sigma}}(\beta) \otimes \widehat{\Omega}_{\mathbb{P}_{\Sigma}}^{p}\right)=0
$$

for $q>p$ or $p>q+\operatorname{dim} P_{\beta}$, where $P_{\beta}$ is the polytope associated with the line bundle $\mathcal{O}_{\mathbb{P}_{\Sigma}}(\beta)$ (see also [7], Theorem 9.3.3). For $p=0$ this reduces to Demazure's vanishing theorem [7, Thm. 9.2.3], that is, $H^{q}\left(\mathbb{P}_{\Sigma}, \mathcal{O}_{\mathbb{P}_{\Sigma}}(\beta)\right)=0$ for $q>0$, under the only hypothesis that $\beta$ is nef.

\section{REgularity AND OdA VARIETIES}

The proofs, based on the infinitesimal variation of Hodge structure, of the NoetherLefschetz theorem, and of the lower bound for the codimension of the Noether-Lefschetz locus in the moduli space of surfaces in $\mathbb{P}^{3}$, use three fundamental ingredients: the (-1)regularity of the hyperplane bundle $\mathcal{O}_{\mathbb{P}^{3}}(1)$, the fact that for any $n, m \in \mathbb{N}$, the multiplication morphism $S_{n} \otimes S_{m} \rightarrow S_{n+m}$ is surjective, and various cohomology vanishings. While these properties do not hold in general for a toric variety, we find that suitable generalizations of the above hypotheses are satisfied by a large class of toric varieties. These generalizations are the key for the proof of Theorem 4.11. 
3.1. Castelnuovo-Mumford regularity for toric varieties. Let $X$ be a projective variety and $L$ an ample and globally generated line bundle on it.

Definition 3.1. A coherent $\mathcal{O}_{X}$-module $\mathcal{F}$ is m-regular with respect to $L$ if

$$
H^{q}\left(X, \mathcal{F} \otimes L^{m-q}\right)=0
$$

for all $q>0$.

If $L$ is an ample and globally generated line bundle which is $m$-regular with respect to itself, we shall just say that it is $m$-regular. A line bundle on a complete toric variety is nef if and only if it is globally generated [18, Th. 1.6], and we have the following:

Theorem 3.2 ([16], Thm 1.8.5). Let $\mathbb{P}_{\Sigma}$ be a projective toric variety. If a locally free $\mathcal{O}_{\mathbb{P}_{\Sigma}}$-module $\mathcal{F}$ is $m$-regular with respect to an ample line bundle $L$, then for all $k \geq 0$,

(i) $\mathcal{F} \otimes L^{m+k}$ is generated by global sections;

(ii) the map

$$
H^{0}\left(\mathbb{P}_{\Sigma}, \mathcal{F} \otimes L^{m}\right) \otimes H^{0}\left(\mathbb{P}_{\Sigma}, L^{k}\right) \rightarrow H^{0}\left(\mathbb{P}_{\Sigma}, \mathcal{F} \otimes L^{k+m}\right)
$$

is surjective;

(iii) $\mathcal{F}$ is $(m+k)$-regular.

Corollary 3.3. If $L$ is 0-regular, for every $m$-regular locally free $\mathcal{O}_{\mathbb{P}_{\Sigma}}$-module $\mathcal{F}$, there is a projective resolution

$$
\cdots \rightarrow \bigoplus\left(L^{-m-1}\right) \rightarrow \bigoplus\left(L^{-m}\right) \rightarrow \mathcal{F} \rightarrow 0
$$

Proof. The proof of [16, Prop. 1.8.8], together with Theorem 3.2, can be applied.

Remark 3.4. Note that $L^{-m-j}$ is $(m+j+1)$-regular if $L$ is 0 -regular, and $(m+j)$-regular if $L$ is $(-1)$-regular.

If $\mathbb{P}_{\Sigma}=\mathbb{P}^{n}$ and $\mathcal{F}$ is locally free and 1-regular with respect to $\mathcal{O}_{\mathbb{P}^{n}}(1)$, then $\mathcal{F}^{\otimes p}$ is $p$ regular a consequence of the fact that $\mathcal{O}_{\mathbb{P}^{n}}(1)$ is $(-1)$-regular [16, Prop. 1.8.9]. The same holds on $\left(\mathbb{P}_{\Sigma}, \eta\right)$ with $\eta$ ample and (-1)-regular:

Proposition 3.5. Let $\mathbb{P}_{\Sigma}$ be a toric variety, and $L$ a $(q-1)$-regular ample line bundle on $i t, q \geq 0$. Il $\mathcal{F}$ is locally free and 1-regular with respect to $L$, then $\mathcal{F}^{\otimes p}$ is $(p+q)$-regular.

Proof. It is enough to show the results when $L$ is $(-1)$ regular. Since $\mathcal{F}$ is locally free, by tensoring $p$ copies of the resolution (3.3) for $m=1$ we obtain a resolution

$$
\cdots \bigoplus\left(L^{-p-1}\right) \rightarrow \bigoplus\left(L^{-p}\right) \rightarrow \mathcal{F}^{\otimes p} \rightarrow 0
$$


By splitting this into short exact sequences and taking cohomology one gets the result.

Proposition 3.6. Let $\mathbb{P}_{\Sigma}$ be an $r$-dimensional toric variety, and $\eta$ an ample Cartier divisor.

(i) $\eta$ is 0-regular if and only if $H^{0}\left(\mathbb{P}_{\Sigma}, \mathcal{O}_{\mathbb{P}_{\Sigma}}\left((r-1) \eta-\beta_{0}\right)\right)=0$.

(ii) $\eta$ is (-1)-regular if and only if $H^{0}\left(\mathbb{P}_{\Sigma}, \mathcal{O}_{\mathbb{P}_{\Sigma}}\left(r \eta-\beta_{0}\right)\right)=0$.

Proof. Follows from Serre duality and the Bott-Danilov-Steenbrink vanishing.

Theorem 3.7. The only Gorenstein toric threefolds with Picard number 1 that carry a 0 -regular ample line bundle are $\mathbb{P}[1,1,2,2]$ and $\mathbb{P}^{3}$.

Proof. Write $\beta_{0}=\ell \eta$, where $\eta$ is an ample Cartier divisor; then $2 \eta-\beta_{0}=(2-\ell) \eta$, and $\eta$ is 0 -regular if and only if $\ell \geq 3$ (Proposition 3.6). Then $\mathbb{P}_{\Sigma}$ is Fano and $\ell$ is the index of $\mathbb{P}_{\Sigma}$ as in [23]. Ogata and Zhao prove that $\ell=3$ if $\mathbb{P}_{\Sigma} \neq \mathbb{P}^{3}[23$, Theorem 1]. The statement then follows from Theorem 3 in [23].

Theorem 3.8. The only $r$-dimensional toric variety $(r \geq 2)$ with an ample line bundle which is (-1)-regular is the projective space $\mathbb{P}^{r}$.

Proof. Let $\eta$ be an ample Cartier divisor in the variety under consideration. By Proposition 3.6(ii) we have that the line bundle $\mathcal{O}_{\mathbb{P}_{\Sigma}}\left(r \eta-\beta_{0}\right)$ has no sections. The claim then follows from Theorem 1 in 23 . (or, for the same statement in purely combinatorial terms, 1 , Prop. 1.4]).

\subsection{Oda varieties.}

Definition 3.9. A toric variety $\mathbb{P}_{\Sigma}$ is an Oda variety if the multiplication morphism $S_{\alpha_{1}} \otimes$ $S_{\alpha_{2}} \rightarrow S_{\alpha_{1}+\alpha_{2}}$ is surjective when the classes $\alpha_{1}$ and $\alpha_{2}$ in $\operatorname{Pic}\left(\mathbb{P}_{\Sigma}\right)$ are ample and nef, respectively.

The question of the surjectivity of this map was posed by Oda in [21] under more general conditions. Note that the conjecture is still open even for smooth projective toric varieties. This assumption can be stated in terms of the Minkowski sum of polytopes, because the integral points of a polytope associated with a line bundle correspond to sections of the line bundle. Definition 3.9 says that the sum $P_{\alpha_{1}}+P_{\alpha_{2}}$ of the polytopes associated with the line bundles $\mathcal{O}_{\mathbb{P}_{\Sigma}}\left(\alpha_{1}\right)$ and $\mathcal{O}_{\mathbb{P}_{\Sigma}}\left(\alpha_{2}\right)$ is equal to their Minkowski sum, that is $P_{\alpha_{1}+\alpha_{2}}$, the polytope associated with the line bundle $\mathcal{O}_{\mathbb{P}_{\Sigma}}\left(\alpha_{1}+\alpha_{2}\right)$. The Oda varieties are characterized by Property 2.2 by Ikeda in [12], as $\alpha_{1}$ is nef if and only if it is globally generated. Ikeda also shows the following: 
Theorem 3.10. [12, Corollary 4.2]

(i) A smooth toric variety with Picard number 2 is an Oda variety.

(ii) The total space of a toric projective bundle on an Oda variety is also an Oda variety.

We have:

Proposition 3.11. Let $\mathbb{P}_{\Sigma}$ be an n-dimensional projective toric variety. If $\operatorname{Pic}\left(\mathbb{P}_{\Sigma}\right)=\mathbb{Z}$ and its ample generator $\eta$ is 0-regular, then $\mathbb{P}_{\Sigma}$ is an Oda variety.

Proof. If $\alpha_{2}$ is a nef class in $\operatorname{Pic}\left(\mathbb{P}_{\Sigma}\right)$, then $\alpha_{2}=\ell \eta$ with $\ell \geq 0$. If $\ell=0$ the condition in Definition 3.9 is empty, so we can assume $\ell \geq 1$. If we set $\mathcal{F}=\mathcal{O}_{\mathbb{P}_{\Sigma}}\left(\alpha_{2}\right)$ and $L=\mathcal{O}_{\mathbb{P}_{\Sigma}}(\eta)$, equation (ii) in Theorem 3.2 becomes the wanted surjectivity condition, provided that $\alpha_{2}$ is 0 -regular (with respect to $\eta$ ). We will show that

$$
H^{i}\left(\mathbb{P}_{\Sigma}, \mathcal{O}_{\mathbb{P}_{\Sigma}}((\ell-i) \eta)\right)=0
$$

for $\ell \geq 1$ and $i>0$. For $0<i<n$ all vanishings are a consequence of the Bott-DanilovSteenbrink vanishing (2.3), possibly using Serre duality (2.3). For $i=n$ and $\ell \geq n$ one uses again the Bott-Danilov-Steenbrink vanishing. For $i=n$ and $\ell=1$ one uses Serre duality and Proposition 3.6, the cases $i=n$ and $1<\ell<n$ follow a fortiori.

3.3. Other vanishings. Let $\mathbb{P}_{\Sigma}=\mathbb{P}^{3}$. Then any ample divisor $\beta$ of degree at least 4 is 0 -regular; also, $\beta-2 H$ is nef. The vanishings $h^{1}\left(\mathbb{P}^{3}, \mathcal{O}_{\mathbb{P}^{3}}(\beta-\eta)\right)=h^{2}\left(\mathbb{P}^{3}, \mathcal{O}_{\mathbb{P}^{3}}(\beta-\eta)\right)=$ $h^{2}\left(\mathbb{P}^{3}, \mathcal{O}_{\mathbb{P}^{3}}(\beta-2 \eta)\right)=0$ follow from either fact. These vanishings are used in the proof of the estimate of the codimensions of the Noether-Lefschetz locus for $\mathbb{P}^{3}$. In our more general setting, the proof of Theorem 4.11 will require analogous vanishing conditions:

$$
h^{1}\left(\mathbb{P}_{\Sigma}, \mathcal{O}_{\mathbb{P}_{\Sigma}}(\beta-\eta)\right)=h^{2}\left(\mathbb{P}_{\Sigma}, \mathcal{O}_{\mathbb{P}_{\Sigma}}(\beta-\eta)\right)=h^{2}\left(\mathbb{P}_{\Sigma}, \mathcal{O}_{\mathbb{P}_{\Sigma}}(\beta-2 \eta)\right)=0 .
$$

So our next step is to study these vanishing conditions.

Proposition 3.12. Let $\mathbb{P}_{\Sigma}$ be a complete simplicial toric variety, $\beta$ and $\eta$ nef divisors. If $\beta-2 \eta$ is nef, then the vanishings in equation (3.3) are satisfied.

Proof. It follows from Demazure's vanishing theorems (the vanishings in (2.3) for $p=$ $0)$.

Proposition 3.13. Let $\mathbb{P}_{\Sigma}$ be a simplicial toric variety, $\beta$ and $\eta$ Cartier divisors, with $\beta$ nef and $\eta$ ample. Assume that $\beta$ is 0-regular with respect to $\eta$. Then:

(i) the vanishings in equation (3.3) are satisfied; 
(ii) the multiplication morphism $S_{\beta} \otimes S_{k \eta} \rightarrow S_{\beta+k \eta}$ is surjective for all $k \geq 0$.

Proof. In fact $\beta$ is also 1-regular by (iii) in Theorem 3.2 and the vanishings follow from the definition of regularity. Part (ii) in Theorem 3.2 implies the surjectivity of the multiplication map.

Corollary 3.14. Let $\mathbb{P}_{\Sigma}$ be a Fano toric simplicial threefold, $\eta$ an ample Cartier class, and $\beta=\beta_{0}+n \eta$. If $n \geq 2$, then $\beta-2 \eta$ is ample. If $n \geq 3$, then $\beta$ is 0-regular with respect to $\eta$.

\section{Noether-Lefschetz LOCI}

In this section we continue the analysis of [4] for $X$ a general surface in a complete simplicial toric variety $\mathbb{P}_{\Sigma}$ corresponding to an ample Cartier divisor; $X$ is quasi-smooth, see Sections 2.1 and 2.2. Let $\mathcal{M}_{\beta}$ be the moduli space of surfaces in $\mathbb{P}_{\Sigma}$ of degree $\beta$ modulo automorphisms of $\mathbb{P}_{\Sigma}$ (for a precise definition see [2]). We want to establish a lower bound for the codimension of the closed subscheme of $\mathcal{M}_{\beta}$ corresponding to surfaces whose Picard number is strictly larger than that of $\mathbb{P}_{\Sigma}$. The codimension will depend on a fixed class $\eta \in \operatorname{Pic}\left(\mathbb{P}_{\Sigma}\right)$, an ample primitive Cartier class (primitive means that it is not a multiple of an ample class). If $\mathbb{P}_{\Sigma}=\mathbb{P}^{3}$ the condition that $D+K_{\mathbb{P}_{\Sigma}}$ is nef in the theorems below is the classical condition $\operatorname{deg}(D) \geq 4$.

Remark 4.1. Note that the condition $\beta-\beta_{0}=n \eta$ implies that the anti-canonical class $\beta_{0}$ is Cartier, namely, $\mathbb{P}_{\Sigma}$ is necessarily Gorenstein.

In [4] we proved:

Theorem 4.2. 4] Let $\mathbb{P}_{\Sigma}$ be a 3-dimensional simplicial toric variety, $D$ an ample Cartier divisor on $\mathbb{P}_{\Sigma}, X$ a very general (quasi-smooth) surface in the linear system $|D|, \beta=\operatorname{deg} D$ and $\beta_{0}=-\operatorname{deg} K_{\mathbb{P}_{\Sigma}}$. If the morphism

$$
R(f)_{\beta} \otimes R(f)_{\beta-\beta_{0}} \rightarrow R(f)_{2 \beta-\beta_{0}}
$$

is surjective, $X$ and $\mathbb{P}_{\Sigma}$ have the same Picard number.

Actually when we wrote [4] we were unaware of [25] where a general result is proved using different techniques. 
Theorem 4.3. Let $\mathbb{P}_{\Sigma}$ be a 3-dimensional simplicial toric Oda variety, $D$ an ample Cartier divisor on $\mathbb{P}_{\Sigma}$ such that $D+K_{\mathbb{P}_{\Sigma}}$ is nef, $X$ a very general (quasi-smooth) surface in the linear system $|D|$. Then $X$ and $\mathbb{P}_{\Sigma}$ have the same Picard number.

Proof. Let $\beta=\operatorname{deg} D, \beta_{0}=-\operatorname{deg} K_{\mathbb{P}_{\Sigma}}$ and note that the multiplication morphism (4.2) is surjective on an Oda variety.

Theorem 4.4. Let $\mathbb{P}_{\Sigma}$ be a 3-dimensional simplicial toric variety, and $D$ an ample Cartier divisor on $\mathbb{P}_{\Sigma}$. Let $\beta=\operatorname{deg} D$, and assume that $\beta=\beta_{0}+n \eta$ with $n \geq 0$ for an ample Cartier class $\eta$. If $\beta$ is 0 -regular with respect to $\eta$ and $X$ is a very general (quasi-smooth) surface in the linear system $|D|$, then $X$ and $\mathbb{P}_{\Sigma}$ have the same Picard number.

Proof. The map (4.2) is surjective because $S_{\beta} \otimes S_{n \eta} \rightarrow S_{\beta+n \eta}$ is by Proposition 3.13 ,

Definition 4.5 (Noether-Lefschetz locus). Let $\mathbb{P}_{\Sigma}$ be a simplicial toric threefold, $\eta$ a primitive ample Cartier class, $\beta_{0}=-K_{\mathbb{P}_{\Sigma}}$ and $\beta \in \operatorname{Pic}\left(\mathbb{P}_{\Sigma}\right)$ an ample Cartier class that satisfies $\beta-\beta_{0}=n \eta$ for some $n \geq 0$. Assume that very general surfaces in the linear system $\beta$ have the same Picard number of $\mathbb{P}_{\Sigma}$.

$U_{\eta}(n)$ is the closed subscheme of $\mathcal{M}_{\beta}$, the moduli space of surfaces in $\mathbb{P}_{\Sigma}$ of degree $\beta$ modulo automorphisms, corresponding to surfaces whose Picard number is strictly larger than that of $\mathbb{P}_{\Sigma}$.

Proposition 4.6. The codimension of any irreducible component of $U_{\eta}(n)$ is bounded from above by $h^{2,0}(S)=h^{0}\left(\mathbb{P}_{\Sigma}, \mathcal{O}_{\mathbb{P}_{\Sigma}}(n \eta)\right)$, where $S$ is a quasi-smooth surface in the linear system $\beta$.

Proof. The classic inequality $\operatorname{codim} U_{\eta}(n) \leq h^{2,0}(S)$ for surfaces in $\mathbb{P}^{3}$ [5, pp. 71-72] relies on the fact that a 2-cycle in a smooth projective surface $S$ is algebraic if and only if it is orthogonal to $H^{2,0}(S)$. In our case, a general surface $S$ in the linear system $\beta$ is quasi-smooth and is a projective orbifold. However the argument may be repeated in this case by taking a resolution of singularities $\rho: S^{\prime} \rightarrow S$. The induced morphism $\rho^{*}: H^{2}(S, \mathbb{C}) \rightarrow H^{2}\left(S^{\prime}, \mathbb{C}\right)$ is injective and the Hodge decomposition of $H^{2}\left(S^{\prime}, \mathbb{C}\right)$ induces a Hodge decomposition of $H^{2}(S, \mathbb{C})$ [28]. A class in $H^{1,1}(S) \cap H^{2}(S, \mathbb{Z})$ regarded in $H^{1,1}\left(S^{\prime}\right) \cap H^{2}\left(S^{\prime}, \mathbb{Z}\right)$ is algebraic and so is algebraic in $S$ as well (the other implication is obvious). To see that $h^{2,0}(S)=h^{0}\left(\mathbb{P}_{\Sigma}, \mathcal{O}_{\mathbb{P}_{\Sigma}}(n \eta)\right)$ one uses the fundamental sequence of the divisor $S$, with Serre duality and the acyclicity of the sheaf $\mathcal{O}_{\mathbb{P}_{\Sigma}}$. Note that the canonical class of $S$ is Cartier by the adjunction formula, since $\beta_{0}$ and $\beta$ are Cartier. 
4.1. Some preliminary Lemmas. To give an estimate on the codimension of $U_{\eta}(n)$ we need some preliminary Lemmas. Let $\mathbb{P}_{\Sigma}$ be a simplicial toric threefold.

In the following we assume that $\eta$ is a primitive ample 0-regular Cartier class, and $\beta \in \operatorname{Pic}\left(\mathbb{P}_{\Sigma}\right)$ a nef Cartier class that satisfies $\beta-\beta_{0}=n \eta$ for some $n \geq 0$, with $\beta_{0}=-K_{\mathbb{P}_{\Sigma}}$. We also assume that the multiplication morphism $S_{\beta} \otimes S_{k \eta} \rightarrow S_{\beta+k \eta}$ is surjective for all $k \geq 0$, and that $h^{1}\left(\mathbb{P}_{\Sigma}, \mathcal{O}_{\mathbb{P}_{\Sigma}}(\beta-\eta)\right)=h^{2}\left(\mathbb{P}_{\Sigma}, \mathcal{O}_{\mathbb{P}_{\Sigma}}(\beta-\eta)\right)=h^{2}\left(\mathbb{P}_{\Sigma}, \mathcal{O}_{\mathbb{P}_{\Sigma}}(\beta-2 \eta)\right)=0$ (see Section 3.3).

Recall that $S_{\beta}=H^{0}\left(\mathbb{P}_{\Sigma}, \mathcal{O}_{\mathbb{P}_{\Sigma}}(\beta)\right)$. Since $\beta$ is nef, it is globally generated, and we have an exact sequence

$$
0 \rightarrow M_{0} \rightarrow S_{\beta} \otimes \mathcal{O}_{\mathbb{P}_{\Sigma}} \rightarrow \mathcal{O}_{\mathbb{P}_{\Sigma}}(\beta) \rightarrow 0
$$

where $M_{0}$ is locally free. Then:

Lemma 4.7. $H^{q}\left(\mathbb{P}_{\Sigma}, M_{0}(k \eta)\right)=0$ for all $q \geq 1$ and $k+q \geq 1$. In particular, $M_{0}$ is 1-regular.

Proof. We twist the exact sequence (4.1) by $\mathcal{O}_{\mathbb{P}_{\Sigma}}(k \eta)$ and take a segment of the long exact sequence of cohomology:

$$
\begin{aligned}
\cdots \rightarrow S_{\beta} \otimes H^{q-1}\left(\mathbb{P}_{\Sigma}, \mathcal{O}_{\mathbb{P}_{\Sigma}}(k \eta)\right) \stackrel{m}{\longrightarrow} & H^{q-1}\left(\mathbb{P}_{\Sigma}, \mathcal{O}_{\mathbb{P}_{\Sigma}}(\beta+k \eta)\right) \\
& \rightarrow H^{q}\left(\mathbb{P}_{\Sigma}, M_{0}(k \eta)\right) \rightarrow S_{\beta} \otimes H^{q}\left(\mathbb{P}_{\Sigma}, \mathcal{O}_{\mathbb{P}_{\Sigma}}(k \eta)\right) \rightarrow \ldots
\end{aligned}
$$

We consider separately the cases $q=1,2,3$.

$q=1$. The morphism $m$ is the multiplication morphism $S_{\beta} \otimes S_{k \eta} \rightarrow S_{\beta+k \eta}$ which is surjective by hypothesis. Moreover $H^{1}\left(\mathbb{P}_{\Sigma}, \mathcal{O}_{\mathbb{P}_{\Sigma}}(k \eta)\right)=0$ by the Demazure vanishing theorem for $k \geq 0$.

$q=2$. If $k \geq 0, H^{1}\left(\mathbb{P}_{\Sigma}, \mathcal{O}_{\mathbb{P}_{\Sigma}}(\beta+k \eta)\right)=H^{2}\left(\mathcal{O}_{\mathbb{P}_{\Sigma}}(k \eta)\right)=0$ by the Demazure vanishing theorem, since $\beta+k \eta$ and $\eta$ are nef. For $k=-1, H^{1}\left(\mathbb{P}_{\Sigma}, \mathcal{O}_{\mathbb{P}_{\Sigma}}(\beta-\eta)\right)=0$ by assumption, and $H^{2}\left(\mathbb{P}_{\Sigma}, \mathcal{O}_{\mathbb{P}_{\Sigma}}(-\eta)=0\right.$ because $\eta$ is 0 -regular.

$q=3$. For $k \geq 0, \beta+k \eta$ is nef so $H^{2}\left(\mathbb{P}_{\Sigma}, \mathcal{O}_{\mathbb{P}_{\Sigma}}(\beta+k \eta)\right)=H^{3}\left(\mathbb{P}_{\Sigma}, \mathcal{O}_{\mathbb{P}_{\Sigma}}(k \eta)\right)=0$ again by Demazure's vanishing. For $k=-2,-1$ we have $H^{2}\left(\mathbb{P}_{\Sigma}, \mathcal{O}_{\mathbb{P}_{\Sigma}}(\beta+k \eta)\right)=0$ by hypothesis and $H^{3}\left(\mathbb{P}_{\Sigma}, \mathcal{O}(k \eta)\right)=0$ because $\eta$ is 0 -regular and by Theorem 3.2 .

Lemma 4.8. Assume that $M_{0}$ is 1-regular. If $\eta$ is (-1)-regular, the vector bundle $\Lambda^{p} M_{0}$ is $p$-regular with respect to $\eta$ for all $p \geq 1$, that is $H^{q}\left(\mathbb{P}_{\Sigma}, \Lambda^{p} M_{0}((p-q) \eta)\right)=0$ for $q>0$.

Similarly, if $\eta$ is 0-regular, then $\Lambda^{p} M_{0}$ is $(p+1)$-regular, that is $H^{q}\left(\mathbb{P}_{\Sigma}, \Lambda^{p} M_{0}((p+1-\right.$ $q) \eta))=0$ for $q>0$. 
Proof. $\Lambda^{p} M_{0}$ is a direct summand of $M_{0}^{\otimes p}$, so that the Lemma follows from Proposition 3.5 .

Let $W$ be a base point free subspace of $S_{\beta}=H^{0}\left(\mathbb{P}_{\Sigma}, \mathcal{O}_{\mathbb{P}_{\Sigma}}(\beta)\right)$, and let

$$
W=W_{c} \subset \ldots \subset W_{0}=S_{\beta}
$$

be a flag of subspaces of $S_{\beta}$ whose quotients $W_{i} / W_{i+1}$ are all of dimension 1 (thus, $c=$ $\left.\operatorname{dim} S_{\beta}-\operatorname{dim} W\right)$. All subspaces $W_{i}$ are base point free (since they contain $W$ ), so that there are exact sequences

$$
0 \rightarrow M_{i} \rightarrow W_{i} \otimes \mathcal{O}_{\mathbb{P}_{\Sigma}} \rightarrow \mathcal{O}_{\mathbb{P}_{\Sigma}}(\beta) \rightarrow 0
$$

with $M_{i}$ locally free. There are injective morphisms $M_{i+1} \rightarrow M_{i}$, and $M_{i} / M_{i+1} \simeq \mathcal{O}_{\mathbb{P}_{\Sigma}}$.

The following Corollary is proved as Lemma 2 of [11].

Corollary 4.9. Assume Lemma 4.8 holds. If $\eta$ is (-1)-regular, $H^{q}\left(\mathbb{P}_{\Sigma}, \Lambda^{p} M_{i}(k \eta)\right)=0$ for where $0 \leq i \leq c=\operatorname{dim} S_{\beta}-\operatorname{dim} W, q \geq 1, p \geq 1, k+q \geq p+i$. If $\eta$ is 0-regular, one has the same vanishing for $q \geq 1, p \geq 1, k+q \geq p+i+1$.

Proposition 4.10. Under the assumption listed at the beginning of Section 4.1, let $W$ be a base point free subspace of $S_{\beta}=H^{0}\left(\mathbb{P}_{\Sigma}, \mathcal{O}_{\mathbb{P}_{\Sigma}}(\beta)\right)$. The map

$$
W \otimes S_{\beta-\beta_{0}} \rightarrow S_{2 \beta-\beta_{0}}
$$

is surjective if $\eta$ is (-1)-regular and codim $W \leq n$.

If $\eta$ is 0-regular but not (-1)-regular, then the map (4.10) is surjective if codim $W \leq n-1$.

Proof. From equation (4.1) for $i=c$ we obtain

$$
W \otimes S_{\beta-\beta_{0}} \rightarrow S_{2 \beta-\beta_{0}} \rightarrow H^{1}\left(\mathbb{P}_{\Sigma}, M_{c}\left(\beta-\beta_{0}\right)\right) .
$$

Since $\beta-\beta_{0}=n \eta$ and $\eta$ is (-1)-regular, the group on the right vanishes by Corollary 4.9 if $n+1 \geq 1+c$, i.e., if codim $W \leq n$ (note indeed that codim $W=c$ ). If $\eta$ is 0 -regular, we obtain the condition codim $W \leq n-1$.

4.2. Codimension of the Noether-Lefschetz loci. We can now prove the lower bounds on the codimension of the Noether-Lefschetz loci.

Theorem 4.11. Let $\mathbb{P}_{\Sigma}$ be a simplicial toric threefold, $\eta$ an ample primitive Cartier class, $\beta_{0}=-K_{\mathbb{P}_{\Sigma}}$ and $\beta \in \operatorname{Pic}\left(\mathbb{P}_{\Sigma}\right)$ an ample Cartier class that satisfies $\beta-\beta_{0}=n \eta$ for some $n \geq 0$. Assume that the multiplication morphism $S_{\beta} \otimes S_{k \eta} \rightarrow S_{\beta+k \eta}$ is surjective and also 
that $h^{1}\left(\mathbb{P}^{3}, \mathcal{O}_{\mathbb{P}^{3}}(\beta-\eta)\right)=h^{2}\left(\mathbb{P}^{3}, \mathcal{O}_{\mathbb{P}^{3}}(\beta-\eta)\right)=h^{2}\left(\mathbb{P}^{3}, \mathcal{O}_{\mathbb{P}^{3}}(\beta-2 \eta)\right)=0$.

If $\eta$ is (-1)-regular, then

$$
\operatorname{codim} U_{\eta}(n) \geq n+1
$$

If $\eta$ is 0-regular, then

$$
\operatorname{codim} U_{\eta}(n) \geq n
$$

Proof. Let $T_{\beta}$ be the tangent space to $U_{\eta}(n)$ at $[X]$. Since $T_{[X]} \mathcal{M}_{\beta}$ may be identified with the summand $R_{\beta}$ of the Jacobian ring of $X[2]$, we may take the inverse image $\tilde{T}_{\beta}$ of $T_{\beta}$ in the summand $S_{\beta}$ of the Cox ring of $X$. Now $\tilde{T}_{\beta}$ contains the summand $J_{\beta}$ of the Jacobian ideal of $X$, which is a base point free linear system because $X$ is quasi-smooth, hence $\tilde{T}_{\beta}$ is base point free as well.

Let us suppose that $\eta$ is $(-1)$-regular, assume that $\operatorname{codim}\left(U_{\eta}(n)\right) \leq n$, and apply Proposition 4.10 by taking $W=\tilde{T}_{\beta}$. The multiplication morphism $\tilde{T}_{\beta} \otimes S_{\beta-\beta_{0}} \rightarrow S_{2 \beta-\beta_{0}}$ is surjective. The proof of Lemma 3.7 in [4] gives that the Picard number of the surfaces in $U_{\eta}(n)$ coincides with the Picard number of $\mathbb{P}_{\Sigma}$. But this contradicts the definition of $U_{\eta}(n)$. The same happens with respect to the condition $\operatorname{codim}\left(U_{\eta}(n)\right) \leq n-1$ when $\eta$ is 0-regular.

Theorem 4.12. Let $\mathbb{P}_{\Sigma}$ be a simplicial toric threefold, $\eta$ an ample primitive Cartier class, $\beta_{0}=-K_{\mathbb{P}_{\Sigma}}, \beta \in \operatorname{Pic}\left(\mathbb{P}_{\Sigma}\right)$ an ample Cartier class that satisfies $\beta-\beta_{0}=n \eta$ for some $n \geq 0$. Assume that $\beta$ is 0-regular with respect to $\eta$. If $\eta$ is (-1)-regular, then

$$
\operatorname{codim} U_{\eta}(n) \geq n+1 \text {. }
$$

If $\eta$ is 0-regular, then

$$
\operatorname{codim} U_{\eta}(n) \geq n
$$

Proof. Follows from Theorem 4.11, Proposition 3.13 and Theorem 4.4.

Corollary 4.13. Let $\mathbb{P}_{\Sigma}$ be a simplicial Fano toric threefold, $\eta$ an ample primitive Cartier class, $\beta_{0}=-K_{\mathbb{P}_{\Sigma}}, \beta \in \operatorname{Pic}\left(\mathbb{P}_{\Sigma}\right)$ a Cartier class that satisfies $\beta-\beta_{0}=n \eta$ for some $n \geq 3$. If $\eta$ is (-1)-regular, then

$$
\operatorname{codim} U_{\eta}(n) \geq n+1
$$


If $\eta$ is 0-regular, then

$$
\operatorname{codim} U_{\eta}(n) \geq n
$$

Proof. By Corollary $3.14 \beta$ is 0-regular, and then the claim follows from Theorem 4.12 .

Remark 4.14. If $n=2$, we need to assume that $\mathbb{P}_{\Sigma}$ is Oda.

Theorem 4.15. Let $\mathbb{P}_{\Sigma}$ be a simplicial toric Oda threefold $\eta$ an ample primitive Cartier class, $\beta_{0}=-K_{\mathbb{P}_{\Sigma}}, \beta \in \operatorname{Pic}\left(\mathbb{P}_{\Sigma}\right)$ an ample Cartier class that satisfies $\beta-\beta_{0}=n \eta$ for some $n \geq 0$. If $\beta-2 \eta$ is nef and $\eta$ is (-1)-regular, then

$$
\operatorname{codim} U_{\eta}(n) \geq n+1 \text {. }
$$

If $\eta$ is 0-regular, then

$$
\operatorname{codim} U_{\eta}(n) \geq n
$$

Proof. Follows from Theorem 4.11 and Proposition 3.12, as the multiplication map is surjective on Oda toric varieties.

For $\mathbb{P}_{\Sigma}=\mathbb{P}^{3}$ and $\eta$ the hyperplane class, the first inequality in the Theorems above reproduces Green's and Voisin's result [10, 11, 29].

4.3. Examples. We discuss examples of toric threefolds for which the results in section 4.2 apply.

Example 4.16. Let $\mathbb{P}_{\Sigma}$ a toric simplicial Gorenstein threefold with nef anti-canonical bundle and $\eta$ an ample Cartier class. Then any class $\beta=n \eta+\beta_{0}$ is $m$-regular with respect to $\eta$ if $n \geq 3-m$. Here we have used the vanishing (2.3). Note that when $\beta$ is 0-regular, by Proposition 3.13 the multiplication morphism $S_{\beta} \otimes S_{k \eta} \rightarrow S_{\beta+k \eta}$ is surjective for $k \geq 0$.

Example 4.17. Let $\widehat{\mathbb{P}}^{3}$ be the projective 3 -space blown-up along a line and $E$ the exceptional divisor. Denote by $\varpi: \widehat{\mathbb{P}}^{3} \rightarrow \mathbb{P}^{3}$ the blow-down morphism. By Ikeda's Theorem $3.10 \widehat{\mathbb{P}}^{3}$ is an Oda simplicial toric variety. The nef cone of $\widehat{\mathbb{P}}^{3}$ is generated by the class $\eta_{1}$, the pullback of a hyperplane in $\mathbb{P}^{3}$, and the class $\eta_{2}=\eta_{1}-E$.

The anti-canonical class is $\beta_{0}=3 \eta_{1}+\eta_{2}$. The class $\eta=\eta_{1}+s \eta_{2}$ is ample and 0 -regular for all $s \geq 1$. The class $\beta-2 \eta$ is nef for $n \geq 2-\frac{1}{s}$. A very general surface $X$ in the linear system of the class $\beta$, with $n \geq 2-\frac{1}{s}$, has Picard number 2 by Theorem 4.3 . 

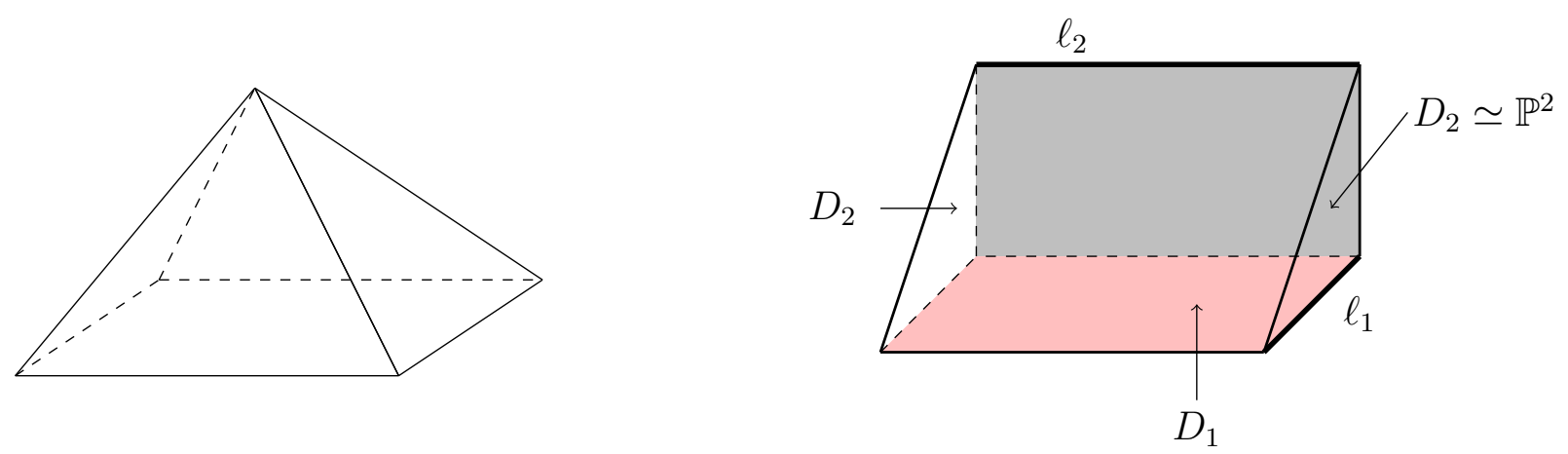

Figure 1. Example 4.19, The picture on the left is the cone over a quadric in $\mathbb{P}^{3}$, the picture on the right is its small resolution, obtained by adding the line $\ell_{2}$. $D_{1}, D_{2}$ are divisors representing the classes $\eta_{1}, \eta_{2}$.

Example 4.18. $\mathbb{P}^{1} \times \mathbb{P}^{2}$ is an Oda variety by Ikeda's Theorem 3.10, The nef cone is generated by the pullback of the hyperplane bundles by the natural projections, namely by the nef classes $H_{1}=\pi_{1}^{*}\left(\mathcal{O}_{\mathbb{P}^{2}}(1)\right)$ and $H_{2}=\pi_{2}^{*}\left(\mathcal{O}_{\mathbb{P}^{1}}(1)\right)$; the nef cone is also the cone of effective divisors.

The ample classes $\eta=H_{1}+s H_{2}, s \geq 1$, are 0-regular. Moreover, $\beta-2 \eta$ is nef if $\beta=\beta_{0}+n \eta$, with $n \geq 2(s-1) / s$. A very general surface $X$ in the linear system $\beta$ has Picard number 2 by Theorem 4.3 .

Example 4.19. Let $\overline{\mathbb{P}}_{\Sigma}$ be the cone over a quadric surface in $\mathbb{P}^{3}$. It is a non-simplicial projective toric threefold [23, §2]. We make a small resolution by adding a face which splits the cone having four edges, getting a smooth simplicial toric threefold $\mathbb{P}_{\Sigma}$. It is easy to see that $\mathbb{P}_{\Sigma}$ is quasi-Fano. Explicit toric computations show that the nef cone is generated by $\eta_{1}$, the pullback of the ample generator of the class group of the quadric surface, and $\eta_{2}$, the divisor giving the fibration over $\mathbb{P}^{1}$ (see Figure 1 ). Moreover, every ample divisor of the form $\eta=\eta_{1}+\eta_{2}$ is 0 -regular. The anti-canonical class is $\beta_{0}=3 \eta_{1}$. By Theorem [3.10, $\mathbb{P}_{\Sigma}$ is an Oda variety. With $\beta=\beta_{0}+n \eta$, the class $\beta-2 \eta$ is nef for $n \geq 2$. Then a very general surface $X$ in the linear system of the class $\beta$ has Picard number 2 by Theorem 4.3, and codim $U_{\eta}(n) \geq n$ by Theorem 4.15.

Example 4.20. Let $\mathbb{P}_{\Sigma}=\mathbb{P}[1,1,2,2]$ and $\eta_{0}$ be the effective generator of the class group of $\mathbb{P}_{\Sigma}$, and let $\eta$ be the (very) ample generator of the Picard group. Note that $\eta=2 \eta_{0}$ and $\beta_{0}=6 \eta_{0}=3 \eta$. The variety $\mathbb{P}_{\Sigma}=\mathbb{P}[1,1,2,2]$ can be realized as a quotient $\mathbb{P}^{3} / \mathbb{Z}_{2}$, and is singular along a toric curve $C$ whose class is $\eta_{0} \cdot \eta_{0}$; this is a line of singularities of type $A_{1}$. 
By Theorem 3.7, $\mathbb{P}_{\Sigma}$ is and Oda variety and $\eta$ is 0 -regular, but not (-1)-regular. Let $\beta=\beta_{0}+n \eta=(3+n) \eta$ with $n \geq 0$; then $\beta-2 \eta$ is nef. A very general surface $X$ in the linear system of the class $\beta$ has Picard number 1 by Theorem 4.3 , and $\operatorname{codim} U_{\eta}(n) \geq n$ by Theorem 4.15,

\section{Components of small Codimension}

5.1. Lines and surfaces containing a line. In the previous Section we have shown that if $\mathbb{P}_{\Sigma}$ is an Oda variety and $\eta$ is (-1)-regular, the inequalities

$$
n+1 \leq \operatorname{codim} U_{\eta}(n) \leq h^{0}\left(\mathbb{P}_{\Sigma}, \mathcal{O}_{\mathbb{P}_{\Sigma}}(n \eta)\right)
$$

hold, while if $\eta$ is only 0 -regular,

$$
n \leq \operatorname{codim} U_{\eta}(n) \leq h^{0}\left(\mathbb{P}_{\Sigma}, \mathcal{O}_{\Sigma}(n \eta)\right) .
$$

In the case of $\mathbb{P}^{3}$ it was conjectured in [10] and proved in [11, 10, 30], that the components of $\mathcal{M}_{\beta}$ of codimension $n+1$ are those whose points correspond to surfaces containing a line. In this section we define a notion of "line" with respect to an ample Cartier class in a toric variety, and study the families of surfaces which contain a line in the Examples of the previous section. We show that such components have also codimension $n+1$.

Definition 5.1. Let $\mathbb{P}_{\Sigma}$ be a simplicial toric threefold, equipped with a 0-regular ample Cartier class $\eta$. A line $L$ in $\mathbb{P}_{\Sigma}$ is a smooth rational curve such that $\eta \cdot L=1$.

A line $L$ is therefore a point in the Hilbert schemes of curves $H i l b^{\mathbb{P}_{\Sigma}}$ in $\mathbb{P}_{\Sigma}$ with Hilbert polynomial $p(k)=k+1$ (with respect to the polarization $\eta$ ); this Hilbert scheme is in general reducible.

Let $\beta=\beta_{0}+n \eta$, with $n \geq 0$. Assume that $\beta$ is ample, and let $\mathcal{S}_{1, \eta}(n)$ be the family of surfaces in $\mathbb{P}_{\Sigma}$ of class $\beta$ that contain a line. For a line $L$ in $\mathbb{P}_{\Sigma}$, denote $\mathcal{J}_{L}$ its ideal sheaf.

Proposition 5.2. Assume that all lines $L$ in $\mathbb{P}_{\Sigma}$ verify the condition $H^{1}\left(\mathbb{P}_{\Sigma}, \mathcal{J}_{L}(\beta)\right)=0$. Then

$$
\operatorname{codim} \mathcal{S}_{1, \eta}(n)=n+1+\beta_{0} \cdot L-\operatorname{dim}_{[L]} \operatorname{Hilb}^{\mathbb{P}_{\Sigma}} .
$$

Proof. Let $\mathcal{S}_{L} \subset H^{0}\left(\mathbb{P}_{\Sigma}, \mathcal{O}_{\mathbb{P}_{\Sigma}}(\beta)\right)$ be the family of surfaces in $\mathbb{P}_{\Sigma}$ of degree $\beta$ that contain a fixed line $L$. From the exact sequence

$$
0 \rightarrow \mathcal{J}_{L}(\beta) \rightarrow \mathcal{O}_{\mathbb{P}_{\Sigma}}(\beta) \rightarrow \mathcal{O}_{L}(\beta) \rightarrow 0,
$$


since $H^{1}\left(\mathbb{P}_{\Sigma}, \mathcal{J}_{L}(\beta)\right)=0$, we see that the codimension of $\mathcal{S}_{L}$ is the dimension of $H^{0}\left(L, \mathcal{O}_{L}(\beta)\right)$. This is

$$
\operatorname{dim} H^{0}\left(L, \mathcal{O}_{L}(\beta)\right)=\operatorname{dim} H^{0}\left(\mathbb{P}^{1}, \mathcal{O}_{\mathbb{P}^{1}}\left(\beta_{0} \cdot L+n\right)\right)=\beta_{0} \cdot L+n+1 .
$$

Now we have to vary the line $L$; the effect of this is to subtract from the result the dimension of the Hilbert scheme of curves with Hilbert polynomial $p(k)=k+1$.

(One can note that the general surface in $\mathcal{S}_{1, \eta}(n)$ does not contain two lines.)

Lemma 5.3. Assume that $L \subset \mathbb{P}_{\Sigma}$ is either in the smooth locus of $\mathbb{P}_{\Sigma}$ or a locally complete intersection and that $h^{1}\left(N_{L / \mathbb{P}_{\Sigma}}\right)=0$. Then

(i) $\operatorname{dim}_{[L]} H_{i l b^{\mathbb{P}_{\Sigma}}}=h^{0}\left(N_{L / \mathbb{P}_{\Sigma}}\right)$.

(ii) If $L$ is in the smooth locus of $\mathbb{P}_{\Sigma}$, then $\operatorname{dim}_{[L]} H i l b^{\mathbb{P}_{\Sigma}}=h^{0}\left(N_{L / \mathbb{P}_{\Sigma}}\right)=\beta_{0} \cdot L$.

Proof. (i) The statement follows from [26, Thm. 4.3.5] and [14, Ch. 1, Thm. 2.8, Lemma 2.12, Prop. 2.14].

(ii) Note that from the exact sequence

$$
0 \rightarrow N_{L / \mathbb{P}_{\Sigma}}^{*} \rightarrow \Omega_{\mathbb{P}_{\Sigma} \mid L}^{1} \rightarrow \Omega_{L}^{1} \rightarrow 0
$$

one has $\operatorname{deg} N_{L / \mathbb{P}_{\Sigma}}=\beta_{0} \cdot L-2$, so that from Riemann-Roch one obtains $h^{0}\left(N_{L / \mathbb{P}_{\Sigma}}\right)=$ $\beta_{0} \cdot L$.

Corollary 5.4. If $\operatorname{dim}_{[L]} H i l b^{\mathbb{P}_{\Sigma}}=\beta_{0} \cdot L$, then

$$
\operatorname{codim} \mathcal{S}_{1, \eta}(n)=n+1 \text {. }
$$

In our examples, the required vanishing $H^{1}\left(\mathbb{P}_{\Sigma}, \mathcal{J}_{L}(\beta)\right)=0$ in Proposition 5.2 will be provided by the following theorem, which generalizes to the case of simplicial toric threefolds a result by Severi [27] and Bertram, Ein and Lazarsfeld [3].

Theorem 5.5. Let $\mathbb{P}_{\Sigma}$ be a simplicial projective toric threefold, and let $L$ be a toric irreducible curve in $\mathbb{P}_{\Sigma}$, which is not contained in the singular locus of $\mathbb{P}_{\Sigma}$, and is the intersection of two effective divisors $D_{1}$ and $D_{2}$, with $D_{1}$ Cartier. Let $\mu: X \rightarrow \mathbb{P}_{\Sigma}$ be the blowup of $\mathbb{P}_{\Sigma}$ along $L$, denote by $Y$ the strict transform of $D_{1}$, and let $D$ be a divisor in $\mathbb{P}_{\Sigma}$ such that

(i) $D-D_{1}$ is nef;

(ii) the line bundle

$$
\mu^{*}\left(\mathcal{O}_{X}\left(D-D_{1}\right)\right)_{\mid Y}+N_{Y / X}
$$

is nef. 
Then $H^{i}\left(\mathbb{P}_{\Sigma}, \mathcal{J}_{L}(D)\right)=0$ for $i>0$.

Proof. One has $\mu_{*}\left(\mathcal{O}_{X}(-F)\right) \simeq \mathcal{J}_{L}$, where $F \subset X$ is the exceptional divisor. We see that one has indeed $\mu_{*} \mathcal{O}_{X} \simeq \mathcal{O}_{\mathbb{P}_{\Sigma}}$, and $R^{i} \mu_{*} \mathcal{O}_{X}=0$ for $i>0$ as $\mu$ is a toric morphism [7, Thm. 9.2.5]. By applying the functor $\mu_{*}$ to the fundamental exact sequence of the divisor $F$, we obtain that $\mu_{*}\left(\mathcal{O}_{X}(-F)\right)$ is a rank 1 subsheaf of $\mathcal{O}_{\mathbb{P}_{\Sigma}}$, i.e., it is the ideal sheaf of a closed subscheme $L^{\prime}$ of $L$. Actually, $L^{\prime}=L$; indeed, if $L^{\prime}$ were properly contained in $L$, it would consist of a finite number of points, and its structure sheaf would be a torsion subsheaf of $\mu_{*} \mathcal{O}_{F \mid L}$. However, the latter is locally free, as follows from the fact that all fibres of $F \rightarrow L$ are integral rational curves, so that $F \rightarrow L$ is a flat morphism, and one can apply cohomology and base change. So $L^{\prime}=L$, and $\mu_{*}\left(\mathcal{O}_{X}(-F)\right) \simeq \mathcal{J}_{L}$.

Then we have the exact sequence

$$
0 \rightarrow \mathcal{J}_{L} \rightarrow \mathcal{O}_{\mathbb{P}_{\Sigma}} \rightarrow \mu_{*} \mathcal{O}_{F} \rightarrow R^{1} \mu_{*}\left(\mathcal{O}_{X}(-F)\right) \rightarrow R^{1} \mu_{*} \mathcal{O}_{X}=0 .
$$

Since the first three terms form an exact sequence, we obtain $R^{1} \mu_{*}\left(\mathcal{O}_{X}(-F)\right)=0$, and similarly $R^{i} \mu_{*}\left(\mathcal{O}_{X}(-F)\right)=0$ for $i>1$. Now the projection formula and the Leray spectral sequence give

$$
H^{i}\left(X, \mu^{*} \mathcal{O}_{\mathbb{P}_{\Sigma}}(D) \otimes \mathcal{O}_{X}(-F)\right) \simeq H^{i}\left(\mathbb{P}_{\Sigma}, \mathcal{J}_{L}(D)\right)
$$

If the divisor $G=\mu^{*}(D)-F$ is nef the conclusion of the Theorem will follow from Demazure's vanishing theorem ([7, Thm. 9.2.3]). Let $\Gamma$ be an irreducible curve in $X$. If $\Gamma$ is not contained in $Y$, then the $[G] \cdot \Gamma \geq 0$ as $D-D_{1}$ is nef. If $\Gamma$ is in $Y$, then $[G] \cdot \Gamma \geq 0$ as the line bundle $($ (ii) $)$ is nef.

We revisit now the Examples in Section 4 and compute the codimension of the loci of surfaces containing a line. In all cases, the vanishing in Theorem 5.5 holds. Moreover, we can apply it also to lines $L$ that are not toric, as a suitable automorphism can always take them to toric lines.

\subsection{Examples: $\mathbb{P}_{\Sigma}$ smooth.}

Example 5.6. We consider the Example 4.17 again. The Mori cone of $\widehat{\mathbb{P}}^{3}$ is generated by the curves $\ell_{1}$ and $\ell_{2}$, where $\ell_{1}$ determines the extremal contraction $\widehat{\mathbb{P}}^{3} \rightarrow \mathbb{P}^{3}$, and $\ell_{2}$ determines the natural morphism $\widehat{\mathbb{P}}^{3} \rightarrow \mathbb{P}^{1}$. One has $\ell_{i} \cdot \eta_{j}=0$ if $i=j$, and $\ell_{i} \cdot \eta_{j}=1$ if $i \neq j$. The class $\eta=\eta_{1}+\eta_{2}$ is very ample, and the curves $\ell_{1}$ and $\ell_{2}$ are lines with respect to it. Note that the Hilbert scheme of curves in $\widehat{\mathbb{P}}^{3}$ with Hilbert polynomial $p(k)=k+1$ has two connected components, corresponding to the homology classes of the lines $\ell_{1}$ and $\ell_{2}$. 
The general line numerically equivalent to $\ell_{2}$ is cut by divisors in the classes $\eta_{1}$ and $\eta_{2}$. It is easy to see that $h^{1}\left(N_{\ell_{2} / \mathbb{P}_{\Sigma}}\right)=0$.

We consider the family $\mathcal{S}_{1, \eta}(n)_{\ell_{2}}$ of surfaces of class $\beta=\beta_{0}+n \eta$ (with $n \geq 0$ ) in $\widehat{\mathbb{P}}^{3}$ that contain a line homologically equivalent to $\ell_{2}$. By Proposition 5.2, Lemma 5.3 and Corollary 5.4

$$
\operatorname{codim} \mathcal{S}_{1, \eta}(n)_{\ell_{2}}=n+1 .
$$

Example 5.7. ( $\mathbb{P}^{1} \times \mathbb{P}^{2}$, Example 4.18) The dual cone of effective curves (the Mori cone) is generated by the rational curves $\ell_{1}=H_{1} \cdot H_{2}$ and $\ell_{2}=H_{1} \cdot H_{1}$. The curves with classes $\ell_{1}$ and $\ell_{2}$ are lines with respect to the ample class $\eta=H_{1}+H_{2}$. It is easy to see that $h^{1}\left(N_{\ell_{1} / \mathbb{P}_{\Sigma}}\right)=h^{1}\left(N_{\ell_{2} / \mathbb{P}_{\Sigma}}\right)=0$. The families of surfaces $\mathcal{S}_{1, \eta}(n)_{\ell_{1}}$ and $\mathcal{S}_{1, \eta}(n)_{\ell_{2}}$ containing a line numerically equivalent to $\ell_{1}$ and $\ell_{2}$, respectively, have both codimension $n+1$ by Proposition 5.2, Lemma 5.3 and Corollary 5.4.

One can note that if we take $\eta=H_{1}+s H_{2}$, then $\mathcal{S}_{1, \eta}(n)_{\ell_{1}}$ has codimension $n s+1$, while $\mathcal{S}_{1, \eta}(n)_{\ell_{2}}$ still has codimension $n+1$ (note indeed that $\ell_{1}$ is not a line).

Example 5.8. (The small resolution of the cone over a quadric, see Example 4.19,) The Mori cone is generated by the lines $\ell_{1}$ and $\ell_{2}$ (see Figure 1 ). $\ell_{2}$ is the exceptional curve of the small resolution, while $\ell_{1}$ determines the natural morphism to $\mathbb{P}^{1}$. Both are lines with respect to the ample class $\eta=\eta_{1}+\eta_{2}$. The general line numerically equivalent to $\ell_{1}$ is cut by divisors in the classes $\eta$ and $\eta_{2}$. It is easy to see that $h^{1}\left(N_{\ell_{1} / \mathbb{P}_{\Sigma}}\right)=0$. If $\beta=\beta_{0}+n \eta$ with $n \geq 2$, we consider the family $\mathcal{S}_{1, \eta}(n)_{\ell_{1}}$ of surfaces in $\mathbb{P}_{\Sigma}$ of class $\beta$ which contain a line homologically equivalent to $\ell_{1}$. Proposition 5.2 , Lemma 5.3 and Corollary 5.4 yield

$$
\operatorname{codim} \mathcal{S}_{1, \eta}(n)_{\ell_{1}}=n+1 .
$$

\section{3. $\mathbb{P}_{\Sigma}=\mathbb{P}[1,1,2,2]$.}

We revisit also Example 4.20, The numerical class of a line $L$ is given by $\eta \cdot \eta_{0}$. We set as usual $\beta=\beta_{0}+n \eta$, with $n \geq 0$. We prove that $\operatorname{dim} H i l b^{\mathbb{P}_{\Sigma}}=\beta_{0} \cdot L=3$, so that the family $\mathcal{S}_{1}$ of surfaces in $\mathbb{P}_{\Sigma}$ that contain a line has codimension $n+1$. Note that since $L$ is a complete intersection, Lemma 5.3 applies if $h^{1}\left(N_{L / \mathbb{P}_{\Sigma}}\right)=0$. In fact we have:

Lemma 5.9. $\operatorname{deg} N_{L / \mathbb{P}_{\Sigma}}=1$ and $h^{1}\left(N_{L / \mathbb{P}_{\Sigma}}\right)=0$.

Proof. We consider the exact sequence

$$
0 \rightarrow K \rightarrow \mathcal{J}_{L} / \mathcal{J}_{L}{ }^{2} \stackrel{d}{\rightarrow} \Omega_{\mathbb{P}_{\Sigma} \mid L}^{1} \rightarrow \Omega_{L}^{1} \rightarrow 0
$$

where $\Omega_{\mathbb{P}_{\Sigma}}^{1}$ is the sheaf of Kähler differentials on $\mathbb{P}_{\Sigma}$. $K$ is a torsion sheaf concentrated at the intersection point $P$ of $L$ with the curve $C$ of singularities of $\mathbb{P}_{\Sigma}$. Moreover, $K$ is the 
torsion of $\mathcal{J}_{L} / \mathcal{J}_{L}{ }^{2}$. Since $L$ is a smooth curve, $\mathcal{J}_{L} / \mathcal{J}_{L}{ }^{2}$ splits as

$$
\mathcal{J}_{L} / \mathcal{J}_{L}^{2}=N_{L / \mathbb{P}_{\Sigma}}^{*} \oplus K,
$$

indeed (see e.g. [26]), by dualizing (5.3) we obtain

$$
0 \rightarrow T_{L} \rightarrow\left(\Omega_{\mathbb{P}_{\Sigma} \mid L}^{1}\right)^{*} \rightarrow N_{L / \mathbb{P}_{\Sigma}} \rightarrow 0 .
$$

Hence we have

$$
\operatorname{deg} N_{L / \mathbb{P}_{\Sigma}}=3-2=1 .
$$

Lemma 5.10. $H^{0}\left(L, \Omega_{\mathbb{P}_{\Sigma} \mid L}^{1}\right)=0$.

Proof. Let $\pi: \mathbb{P}^{3} \rightarrow \mathbb{P}^{3} / \mathbb{Z}_{2}=\mathbb{P}_{\Sigma}$. From [8, 13] we have

$$
\pi_{*}^{\mathbb{Z}_{2}} \Omega_{\mathbb{P}^{3}}^{1} \simeq \widehat{\Omega}_{\mathbb{P}_{\Sigma}}^{1}
$$

where $\widehat{\Omega}_{\mathbb{P}_{\Sigma}}^{1}$ is the sheaf of Zariski differentials (see section 2.3 ). Let $\tilde{L}=\pi^{-1}(L)$. Note that $H^{0}\left(\tilde{L}, \Omega_{\mathbb{P}^{3} \mid \tilde{L}}^{1}\right)=0$, as follows from the Euler sequence of $\mathbb{P}^{3}$ restricted to $\tilde{L}$. Also, we have

$$
H^{0}\left(L, \widehat{\Omega}_{\mathbb{P}_{\Sigma} \mid L}^{1}\right)=H^{0}\left(L, \pi_{*}^{\mathbb{Z}_{2}} \Omega_{\mathbb{P}^{3} \mid \tilde{L}}^{1}\right)=H^{0}\left(\tilde{L}, \Omega_{\mathbb{P}^{3}}^{1}\right)^{\mathbb{Z}_{2}} .
$$

Since $H^{0}\left(\tilde{L}, \Omega_{\mathbb{P}^{3}}^{1}\right)^{\mathbb{Z}_{2}}=0$, we have

$$
H^{0}\left(L, \widehat{\Omega}_{\mathbb{P}_{\Sigma} \mid L}^{1}\right)=0
$$

Let $i: U \rightarrow \mathbb{P}_{\Sigma}$ be the embedding of the smooth locus of $\mathbb{P}_{\Sigma}$. By definition, $\widehat{\Omega}_{\mathbb{P}_{\Sigma}}^{1}=$ $i_{*} i^{*} \Omega_{\mathbb{P}_{\Sigma}}^{1}$, so that we have an exact sequence

$$
0 \rightarrow J \rightarrow \Omega_{\mathbb{P}_{\Sigma}}^{1} \rightarrow \widehat{\Omega}_{\mathbb{P}_{\Sigma}}^{1} \rightarrow Q \rightarrow 0 .
$$

However, since $J$ is supported on the singular locus of $\mathbb{P}_{\Sigma}$, and $\Omega_{\mathbb{P}_{\Sigma}}^{1}$ is torsion-free one has $J=0$ [15, Prop. 9.7 and Cor. 9.8], [13, Thm. 3] (see also [28, Lemma 1.8] and [9]). The sheaf $Q$ is supported on the singular locus as well. By restricting to $L$ we obtain

$$
\operatorname{Tor}_{1}\left(Q, \mathcal{O}_{L}\right) \rightarrow \Omega_{\mathbb{P}_{\Sigma} \mid L}^{1} \rightarrow \widehat{\Omega}_{\mathbb{P}_{\Sigma} \mid L}^{1}
$$

Now $\operatorname{Tor}_{1}\left(Q, \mathcal{O}_{L}\right)$ is concentrated on $P$, and since $\Omega_{\mathbb{P}_{\Sigma} \mid L}^{1}$ is an extension of the locally free sheaves $N_{L / \mathbb{P}_{\Sigma}}^{*}$ and $\Omega_{L}^{1}$, it is locally free as well, hence $\operatorname{Tor}_{1}\left(Q, \mathcal{O}_{L}\right)$ maps to zero, and $\Omega_{\mathbb{P}_{\Sigma} \mid L}^{1}$ injects into $\widehat{\Omega}_{\mathbb{P}_{\Sigma} \mid L}^{1}$. From (5.3) we get

$$
H^{0}\left(L, \Omega_{\mathbb{P}_{\Sigma} \mid L}^{1}\right)=0 .
$$


Now we consider the exact sequence

$$
0 \rightarrow N_{L / \mathbb{P}_{\Sigma}}^{*}(-2) \rightarrow \Omega_{\mathbb{P}_{\Sigma} \mid L}^{1}(-2) \rightarrow \mathcal{O}_{L}(-4) \rightarrow 0
$$

whence we get $h^{0}\left(N_{L / \mathbb{P}_{\Sigma}}^{*}(-2)\right)=0$. By Serre duality, $h^{1}\left(N_{L / \mathbb{P}_{\Sigma}}\right)=0$, so that

$$
h^{0}\left(N_{L / \mathbb{P}_{\Sigma}}\right)=\chi\left(N_{L / \mathbb{P}_{\Sigma}}\right)=3 .
$$

So also in this case we have shown that the family $\mathcal{S}_{1, \eta}(n)_{L}$ has codimension $n+1$.

\section{REFERENCES}

[1] V. Batyrev And B. NiLl, Multiples of lattice polytopes without interior lattice points, Mosc. Math. J., 7 (2007), pp. 195-207, 349.

[2] V. V. Batyrev and D. A. Cox, On the Hodge structure of projective hypersurfaces in toric varieties, Duke Math. J., 75 (1994), pp. 293-338.

[3] A. Bertram, L. Ein, and R. Lazarsfeld, Vanishing theorems, a theorem of Severi, and the equations defining projective varieties, J. Amer. Math. Soc., 4 (1991), pp. 587-602.

[4] U. Bruzzo and A. Grassi, Picard group of hypersurfaces in toric varieties, Int. J. Math., 23 (2012). No. 2, 1250028.

[5] J. Carlson, M. Green, P. Griffiths, and J. Harris, Infinitesimal variations of Hodge structure. I, Compositio Math., 50 (1983), pp. 109-205.

[6] D. A. Cox, The homogeneous coordinate ring of a toric variety, J. Algebraic Geom., 4 (1995), pp. 1750.

[7] D. A. Cox, J. B. Little, And H. K. Schenck, Toric varieties, vol. 124 of Graduate Studies in Mathematics, American Mathematical Society, Providence, RI, 2011.

[8] I. Dolgachev, Weighted projective varieties, in Group actions and vector fields (Vancouver, B.C., 1981), vol. 956 of Lecture Notes in Math., Springer, Berlin, 1982, pp. 34-71.

[9] D. Greb And S. Rollenske, Torsion and cotorsion in the sheaf of Kähler differentials on some mild singularities, Math. Res. Lett., 18 (2011), pp. 1259-1269.

[10] M. L. Green, Koszul cohomology and the geometry of projective varieties. II, J. Differential Geometry, 20 (1984), pp. 279-289.

[11] — A new proof of the explicit Noether-Lefschetz theorem, J. Differential Geometry, 27 (1988), pp. $155-159$.

[12] A. IKEDA, Subvarieties of generic hypersurfaces in a nonsingular projective toric variety, Math. Z., 263 (2009), pp. 923-937.

[13] C. M. Knighten, Differentials on quotients of algebraic varieties, Trans. Amer. Math. Soc., 177 (1973), pp. 65-89.

[14] J. Kollár, Rational curves on algebraic varieties, vol. 32 of Ergebnisse der Mathematik und ihrer Grenzgebiete. 3. Folge. A Series of Modern Surveys in Mathematics, Springer-Verlag, Berlin, 1996.

[15] E. Kunz, Kähler differentials, Advanced Lectures in Mathematics, Friedr. Vieweg \& Sohn, Braunschweig, 1986. 
[16] R. Lazarsfeld, Positivity in algebraic geometry. I, vol. 48 of Ergebnisse der Mathematik und ihrer Grenzgebiete (3), Springer-Verlag, Berlin, 2004. Classical setting: line bundles and linear series.

[17] A. F. Lopez and C. Maclean, Explicit Noether-Lefschetz for arbitrary threefolds, Math. Proc. Cambridge Philos. Soc., 143 (2007), pp. 323-342.

[18] A. R. Mavlyutov, Semiample hypersurfaces in toric varieties, Duke Math. J., 101 (2000), pp. 85116.

[19] — Cohomology of rational forms and a vanishing theorem on toric varieties, J. Reine Angew. Math., 615 (2008), pp. 45-58.

[20] J. P. Mullet, Toric Calabi-Yau hypersurfaces fibered by weighted K3 hypersurfaces, Comm. Anal. Geom., 17 (2009), pp. 107-138.

[21] T. ODA, Problems on Minkowski sums of convex lattice polytopes. 0812.1418 [math.AG].

$[22]-$ Convex bodies and algebraic geometry. An introduction to the theory of toric varieties, vol. 15 of Ergebnisse der Mathematik und ihrer Grenzgebiete (3), Springer-Verlag, Berlin, 1988.

[23] S. Ogata And H.-L. ZhaO, A characterization of Gorenstein toric Fano n-folds with index $n$ and Fujita's conjecture. arXiv:1404.6870.

[24] A. Otwinowska, Composantes de petite codimension du lieu de Noether-Lefschetz: un argument asymptotique en faveur de la conjecture de Hodge pour les hypersurfaces, J. Algebraic Geom., 12 (2003), pp. 307-320.

[25] G. V. Ravindra And V. SRInivas, The Noether-Lefschetz theorem for the divisor class group, J. Algebra, 322 (2009), pp. 3373-3391.

[26] E. Sernesi, Deformations of algebraic schemes, vol. 334 of Grundlehren der Mathematischen Wissenschaften [Fundamental Principles of Mathematical Sciences], Springer-Verlag, Berlin, 2006.

[27] F. Severi, Su alcune questioni di postulazione, Rend. Circ. Mat. Palermo, 17 (1903), pp. 73-103.

[28] J. H. M. Steenbrink, Mixed Hodge structure on the vanishing cohomology, in Real and complex singularities (Proc. Ninth Nordic Summer School/NAVF Sympos. Math., Oslo, 1976), Sijthoff and Noordhoff, Alphen aan den Rijn, 1977, pp. 525-563.

[29] C. Voisin, Une précision concernant le théorème de Noether, Math. Ann., 280 (1988), pp. 605-611.

[30] _ Composantes de petite codimension du lieu de Noether-Lefschetz, Comment. Math. Helv., 64 (1989), pp. 515-526. 